University of Wollongong

Research Online

Faculty of Engineering - Papers (Archive)

Faculty of Engineering and Information

Sciences

August 2005

\title{
Enhanced Stiffness Modeling, Identification and Characterization for Robot Manipulators
}

G. Alici

University of Wollongong, gursel@uow.edu.au

B. Shirinzadeh

Monash University

Follow this and additional works at: https://ro.uow.edu.au/engpapers

Part of the Engineering Commons

https://ro.uow.edu.au/engpapers/24

\section{Recommended Citation}

Alici, G. and Shirinzadeh, B.: Enhanced Stiffness Modeling, Identification and Characterization for Robot Manipulators 2005.

https://ro.uow.edu.au/engpapers/24

Research Online is the open access institutional repository for the University of Wollongong. For further information contact the UOW Library: research-pubs@uow.edu.au 


\title{
Enhanced Stiffness Modeling, Identification and Characterization for Robot Manipulators
}

\author{
Gürsel Alici and Bijan Shirinzadeh
}

\begin{abstract}
This paper presents the enhanced stiffness modeling and analysis of robot manipulators, and a methodology for their stiffness identification and characterization. Assuming that the manipulator links are infinitely stiff, the enhanced stiffness model contains: 1) the passive and active stiffness of the joints and 2) the active stiffness created by the change in the manipulator configuration, and by external force vector acting upon the manipulator end point. The stiffness formulation not accounting for the latter is known as conventional stiffness formulation, which is obviously not complete and is valid only when: 1) the manipulator is in an unloaded quasistatic configuration and 2) the manipulator Jacobian matrix is constant throughout the workspace. The experimental system considered in this study is a Motoman SK 120 robot manipulator with a closed-chain mechanism. While the deflection of the manipulator end point under a range of external forces is provided by a high precision laser measurement system, a wrist force/torque sensor measures the external forces. Based on the experimental data and the enhanced stiffness model, the joint stiffness values are first identified. These stiffness values are then used to prove that conventional stiffness modeling is incomplete. Finally, they are employed to characterize stiffness properties of the robot manipulator. It has been found that although the component of the stiffness matrix differentiating the enhanced stiffness model from the conventional one is not always positive definite, the resulting stiffness matrix can still be positive definite. This follows that stability of the stiffness matrix is not influenced by this stiffness component. This study contributes to the previously reported work from the point of view of using the enhanced stiffness model for stiffness identification, verification and characterization, and of new experimental results proving that the conventional stiffness matrix is not complete and is valid under certain assumptions.
\end{abstract}

Index Terms-Compliance/force control, manipulator kinematic, stiffness identification, stiffness modeling.

\section{INTRODUCTION}

$\mathbf{K}$ NOWING the stiffness or compliance of a robot manipulator reflected at its end point is of prime importance to successfully conduct contact and noncontact tasks. In fact, the stiffness of robot manipulators generally represents the accuracy required to satisfy the desired position and force commands [1]-[3]. Further, it must be recalled that there is

Manuscript received May 4, 2004; revised August 4, 2004. This paper was recommended for publication by Associate Editor R. Roberts and Editor I. Walker upon evaluation of the reviewers' comments. This work was supported in part by the Australian Research Council (ARC) and Monash University (SMURF2).

G. Alici is with the School of Mechanical, Materials and Mechatronics Engineering, University of Wollongong, Wollongong, NSW 2522, Australia (e-mail: gursel@uow.edu.au).

B. Shirinzadeh is with Robotics and Mechatronics Research Laboratory, Department of Mechanical Engineering, Monash University, Melbourne, Victoria 3800, Australia (e-mail: bijan.shirinzadeh@eng.monash.edu.au).

Digital Object Identifier 10.1109/TRO.2004.842347 coupling among rotational and translational Cartesian movements of a typical serially connected robot manipulator because of the inconsistency between the task specification (Cartesian) space and the actuation (joint) space. This coupling also shows itself in the nondiagonal stiffness matrix seen at the end point of the robot manipulator. As a result, the manipulator tool frame rotates when an end point force is applied along one of the degrees of freedom [1], [2]. If the stiffness seen at the manipulator end point is modeled and identified accurately, it would be possible to compensate for the coupling and posing errors caused by the external forces. The model may also be used to generate manipulator stiffness maps defining the manipulator end point stiffness as functions of the joint stiffness and manipulator configurations. The minimum and maximum stiffness values, which are the maximum and minimum eigenvalues of the stiffness matrix, and their directions, which are the corresponding eigenvectors, can be known in advance. Hence, the most appropriate configurations for certain tasks can be selected. In this study, we, therefore, address enhanced stiffness modeling, analysis, identification and characterization for robot manipulators. The enhanced stiffness model differs from the conventional stiffness model first derived by Mason and Salisbury [4] such that it contains the stiffness component due to the change in the manipulator configuration and the external forces acting on the manipulator in addition to the inherent stiffness of the system. The conventional stiffness formulation is valid only when the manipulator is in a quasistatic configuration with no loading, or when it has a constant Jacobian matrix throughout its workspace such as a Cartesian robot manipulator. Although the existence of the additional stiffness component has been known for a long time [5], [6], its importance for preserving the conservative and fundamental properties of joint and Cartesian stiffness matrices has been highlighted by Kao et al. [9] and others [7], [8], and [10] recently.

Stiffness modeling, analysis, synthesis and control have attracted the attention of many researchers. Ang and Andeen [11] reported on how to generate variable passive compliance through the topology of robot manipulators with backdrivable actuators. It has been concluded that a nondiagonal stiffness matrix can be effective in preventing jamming and contact induced vibrations. Ang et al. [12] also introduced a methodology to model the end-effector stiffness due to link flexibilities of serially connected robot manipulators. They demonstrated that the methodology could be applied to all possible serial manipulator topologies: 1) to describe their end-effector stiffness as a function of manipulator configurations, and 2) to choose the most appropriate manipulator configuration compatible with the compliance requirements of the task at hand. Kao et al. 
[13] have pointed out that the linear $3 \times 3$ stiffness matrices for grasping can be decomposed into symmetric and nonsymmetric components such that they provide better physical insights into stiffness control and grasping. The stiffness components are calibrated using experimental data from some grasping tasks. It has been concluded that the stiffness matrix has a significant effect on grasp stability and robustness against slipping. Hang and Schimmels [14] demonstrated that spatial compliance behavior could be accomplished using a number of springs connected in parallel to a rigid body. In a study on global stiffness modeling of compliant couplings, Griffis and Duffy [15] pointed out that a $6 \times 6$ general stiffness matrix was not symmetric for a conservative system. Ciblak and Lipkin [16] later demonstrated that the skew-symmetric part of such a matrix was due to the externally applied force/moment vectors. Ciblak and Lipkin [17] also employed the spatial vector (screw) algebra to demonstrate that the Cartesian stiffness of robotic systems could be realized by a number of springs. Chen and Kao [7] have recently reported that the conventional stiffness formulation derived by Mason and Salisbury [4] is not valid and a new conservative congruence transformation must be used as the generalized relationship between the joint and Cartesian stiffness matrices in order to preserve the fundamental properties of the stiffness matrices. Based on this finding, a number of publications on the use of this new transformation have been made available by the same group of researchers [7]-[10], simulation results for a planar revolute-jointed manipulator have been provided to demonstrate its validity. With this in mind, it is believed that the work presented in this paper is the first to verify enhanced stiffness modeling through experimental results provided by a high-resolution laser-based position sensing and measurement system.

The sources of the stiffness of a typical robot manipulator are the compliance of its joints, actuators and other transmission elements, geometric and material properties of the links, base, and the active stiffness provided by its position control system. For the purpose of this study, we assume that: 1) the compliance in actuators and transmission elements is the dominant source of the stiffness, and it can be represented by a linear torsional spring for each joint; 2) the active compliance in actuators due to a robot position control system provided by the original equipment manufacturer does not vary with time though an integral controller can increase the active compliance, depending on the positioning error; and 3) the links are infinitely stiff. The experimental system employed here in this work is a Motoman SK 120 robot manipulator, which is not a simple open chain robot manipulator, but rather contains a parallel five-bar mechanism to increase the structural stiffness of the system [18]. In order to identify the joint stiffness values, a number of manipulator configurations are heuristically selected to acquire experimental position and force data, which are provided by a laser-based sensing and measurement system, and a wrist force/torque $(\mathrm{F} / \mathrm{T})$ sensor, respectively. The laser-based sensing system has an accuracy of $\mp 10 \mathrm{ppm}(\mu \mathrm{m} / \mathrm{m})$, a coordinate repeatability of $\mp 5 \mathrm{ppm}(\mu \mathrm{m} / \mathrm{m})$ and a distance resolution of $1.26 \mu \mathrm{m}$, and can measure the position of any target along the three orthogonal axes. A classical nonlinear least square estimation algorithm is used to estimate the joint stiffness values. The estimated joint stiffness matrix is verified experimentally. Also, the work done in Cartesian and joint spaces are computed for both enhanced and conventional stiffness formulations. It has been found that the conventional stiffness formulation does not satisfy the principle of the conservation of energy. It has also been demonstrated that this arises from the existence of the additional stiffness component. Further, based on the estimated joint stiffness constants, the limits and conditions of the positive definiteness (stability) of the Cartesian stiffness matrix are evaluated. It has been found that although the additional stiffness component differentiating the enhanced formulation from the conventional formulation is not always positive definite, the resulting Cartesian stiffness matrix can still be positive definite. The main contribution of this study is the new theoretical and experimental results supporting the enhanced stiffness modeling (or conservative congruence transformation) through stiffness identification, verification, and characterization.

\section{KINEMATIC ANALYSES AND JACOBIAN MATRIX}

The schematic of the robot manipulator and the coordinate frames needed to generate a kinematic model based on Denavit-Hartenberg parameters is depicted in Fig. 1. The manipulator possesses a parallel five-bar mechanism. Therefore, the kinematic model of the parallel five-bar mechanism is also derived. The Denavit Hartenberg parameters for the manipulator and the parallel mechanism are given in Table I.

An L-shaped apparatus with a longitudinal extension of $a=$ $112.65 \mathrm{~mm}$ from the manipulator tool plate and a vertical offset of $b=205.32 \mathrm{~mm}$ from the longitudinal axis of the tool plate is connected to the manipulator tool plate in order to secure the retroreflector of the measurement system to the robot via a 3-point-contact magnetic fixture. With reference to Fig. 1, it must be noted that, for the five-bar mechanism, $\bar{\theta}_{1}=\theta_{3}, \bar{\theta}_{2}=$ $\theta_{2}-\theta_{3}, \bar{\theta}_{2}+\bar{\theta}_{3}=\pi, \bar{L}_{1}=\bar{L}_{3}$, and $\bar{L}_{2}=L_{2}$. The homogeneous transformation matrix between the frames 1 and 2 of the five bar is described in terms of the coordinate frames fixed to the links of the five-bar mechanism as

$$
{ }^{1} T_{2}=\left({ }^{1} T_{\overline{1}}\right)\left({ }^{\overline{1}} T_{\overline{2}}\right)\left({ }^{\overline{2}} T_{\overline{3}}\right)
$$

The overall transformation matrix between the base coordinate frame and the frame fixed to the manipulator end point is written as

$$
{ }^{0} T_{6}=\left({ }^{0} T_{1}\right)\left({ }^{1} T_{2}\right)\left({ }^{5} T_{3}\right)\left({ }^{3} T_{4}\right)\left({ }^{4} T_{5}\right)\left({ }^{5} T_{6}\right)
$$

where ${ }^{j} T_{j+1}$ is the homogeneous transformation matrix between two consecutive coordinate frames $\mathrm{j}$ and $j+1$ based on Denavit-Hartenberg convention [18]. From the first three elements of the last column of ${ }^{0} T_{6}$, the generalized relationship between the input velocity vector $\dot{\Theta}=\left[\dot{\theta}_{1}, \dot{\theta}_{2}, \dot{\theta}_{3}\right]^{T}$ and the output velocity vector $\dot{X}=[\dot{x}, \dot{y}, \dot{z}]^{T}$ is obtained as

$$
\dot{X}=\left[\begin{array}{lll}
\frac{\partial x}{\partial\left(\theta_{1}, \theta_{2}, \theta_{3}\right)} & \frac{\partial y}{\partial\left(\theta_{1}, \theta_{2}, \theta_{3}\right)} & \frac{\partial z}{\partial\left(\theta_{1}, \theta_{2}, \theta_{3}\right)}
\end{array}\right]^{T} \dot{\Theta}=J \dot{\Theta}
$$

where $J$ is the manipulator Jacobian matrix, see Appendix I for analytical expressions of its elements. 


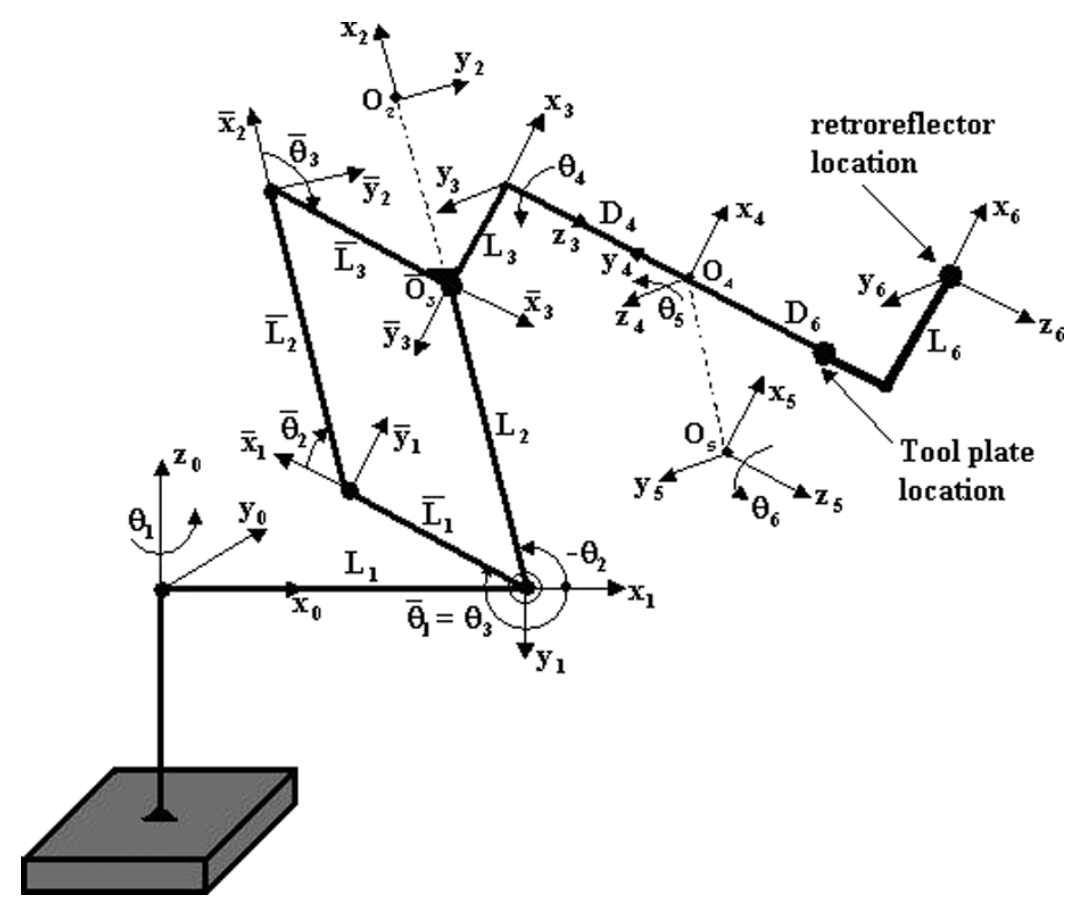

Fig. 1. Schematic representation of the robot manipulator with D-H convention and parameters. Note that the coordinate frames $\mathrm{O}_{2}$ and $\overline{\mathrm{O}}_{3}$, and $\mathrm{O}_{4}$ and $\mathrm{O}_{5}$ are located at the same point.

TABLE I

RESULTS OF D-H PARAMETERS FOR MOTOMAN 120 SK MANIPULATOR AND Parallel MechanisM. SHADEd Rows ARE FOR PARALlEL MECHANISM

\begin{tabular}{l|l|l|l|l}
\hline $\begin{array}{l}\text { Joint } \\
\text { Link }\end{array}$ & $\begin{array}{l}\alpha_{\mathrm{i}} \\
(\text { degree })\end{array}$ & $\begin{array}{l}\mathrm{D}_{\mathrm{i}} \\
(\mathrm{mm})\end{array}$ & $\begin{array}{l}\mathrm{L}_{\mathrm{i}} \\
(\mathrm{mm})\end{array}$ & $\begin{array}{l}\theta_{\mathrm{i}} \\
(\text { degree })\end{array}$ \\
\hline$\overline{1}$ & $-90^{\circ}$ & 0 & 215 & $\theta_{1}$ \\
\hline$\overline{1}$ & 0 & 0 & 542 & $\bar{\theta}_{1}$ \\
\hline$\overline{2}$ & 0 & 0 & 1100 & $\bar{\theta}_{2}$ \\
\hline$\overline{3}$ & 0 & 0 & 542 & $\bar{\theta}_{3}$ \\
\hline 3 & $-90^{\circ}$ & 0 & 250 & $\bar{\theta}_{4}=-90^{\circ}$ \\
\hline 4 & $-90^{\circ}$ & 1300 & 0 & $\theta_{4}$ \\
\hline 5 & $90^{\circ}$ & 0 & 0 & $\theta_{5}$ \\
\hline 6 & 0 & 230 & 0 & $\theta_{6}$ \\
\hline
\end{tabular}

Using the duality between the generalized relationships for motion and force transfer between the actuator and operation spaces, the following force relationship is obtained:

$$
\Gamma=J^{T} F
$$

where $F=\left[F_{x} F_{y}, F_{z}\right]^{T}$ represents the $3 \times 1$ vector of external forces acting at the manipulator end point, and $\Gamma=\left[\tau_{1}, \tau_{2}, \tau_{3}\right]^{T}$ denotes the $3 \times 1$ vector of the actuator forces/torques needed to balance the external forces, and $(.)^{T}$ denotes transposition.

\section{STIFFNESS FORMULATION}

The sources of stiffness for a typical serially connected robot manipulator include the base stiffness, joints' stiffness, link stiffness, and active stiffness due to position feedback. It is assumed that the primary source of the stiffness is the active and passive joint stiffness in the axial direction of the actuation torque, and it is lumped into a single constant stiffness value $K_{\theta i}$ for each joint. However, it must be noted that, depending on positioning error and the integral gain that is usually much smaller than the proportional gain in a typical proportional integral derivative (PID) controller, the active stiffness due to a robot position control system provided by the original equipment manufacturer can vary with time. In this study, for the sake of simplicity without loosing generality, it is assumed that this variation is negligibly small. The actuator force/torque needed to cause a change $\Delta \Theta$ in the angular position of the joints from an unloaded position is

$$
\Gamma=K_{\Theta} \Delta \Theta
$$

where $K_{\Theta}=\operatorname{diag}$. $\left[K_{\theta 1}, K_{\theta 2}, K_{\theta 3}\right]$ is the joint stiffness matrix, and $\Delta \Theta=\left[\delta \theta_{1}, \delta \theta_{2}, \delta \theta_{3}\right]^{T}$ is the three-dimensional vector of the change in the joints position. It must be noted that such a matrix is positive definite and symmetric. Similarly, the force $F$ needed to cause the manipulator end point to experience a small change $\Delta X$ in its position is given by

$$
F=K_{X} \Delta X
$$

where $K_{x}$ is a symmetric $3 \times 3$ matrix representing the Cartesian stiffness of the manipulator, and $\Delta X=[\delta x, \delta y, \delta z]^{T}$ is the vector of the change in the manipulator end point position. The partial differentiation of (4) with respect to $\Theta$ leads to the following relationship:

$$
\underbrace{\frac{\partial \Gamma}{\partial \Theta}}_{K_{\Theta}}=\left(\frac{\partial J^{T}}{\partial \Theta}\right) F+J^{T} \underbrace{\frac{\partial F}{\partial X}}_{K_{X}} \underbrace{\frac{\partial X}{\partial \Theta}}_{J} \Rightarrow K_{\Theta}=\left(\frac{\partial J^{T}}{\partial \Theta}\right) F+J^{T} K_{X} J .
$$

For the static case of unloaded manipulator configuration, the first term on the right-hand side of (7) is zero, and the stiffness 
$K_{X}$ seen at the end point of the manipulator can be expressed as

$$
K_{X}=J^{-T} K_{\Theta} J^{-1}
$$

Equation (8) is the formulation of active stiffness control [4], which regulates the apparent stiffness of a manipulator end point in order to control the nominal position of the end point. Stiffness values are changed in the software to satisfy the desired position commands. It must be noted that $K_{X}$ calculated from (8) is the Cartesian stiffness matrix based on the conventional stiffness formulation, which neglects the stiffness component $K_{C}$ due to external loading and change in the manipulator configuration.

If any positive definite and symmetric matrix $D$ is subjected to a transformation operation in the form of $\left(A^{T}\right) D(A)$, the resulting matrix will still be positive definite and symmetric, as long as the transformation matrix $A$ is nonsingular [19]. This means that the stiffness matrix $K_{x}$ evaluated from (8) is positive definite and symmetric. As provided in [7], [20], the matrix resulting from the first part of (7) is symmetric, but depending on the external forces/payloads and the configuration of the manipulator, it can be positive definite or not. With regard to the second part of (7), the fundamental properties of $K_{x}$ such as its definiteness and symmetry are preserved provided that the Jacobian matrix $\mathbf{J}$ is not singular. With this in mind, (7) always gives a symmetric stiffness matrix $K_{\theta}$, but not necessarily a positive definite $K_{\theta}$. However, as explained above, the joint stiffness matrix is the natural entity of a robot manipulator and does not change with the manipulator configuration. Further, it is a diagonal and positive definite matrix. Therefore, the fundamental properties of the Cartesian stiffness matrix can be evaluated for any existing robot manipulator.

\section{ESTIMATION OF JOINT STIFFNESS}

The enhanced stiffness formulation given by (7) differs from the conventional stiffness formulation in the sense that its first part stands for the case when the manipulator is externally loaded and/or the manipulator Jacobian changes with its configuration. This part of (7) completes the conventional joint stiffness matrix and therefore, we call it the complementary stiffness matrix $K_{C}$, and for a manipulator actuated through three joints, it can be written as

$$
K_{C}=\left[\begin{array}{lll}
\frac{\partial J^{T}}{\partial \theta_{1}} F & \frac{\partial J^{T}}{\partial \theta_{2}} F & \frac{\partial J^{T}}{\partial \theta_{3}} F
\end{array}\right]
$$

where $\left(\partial J^{T} / \partial \theta_{i}\right) F$ is a $3 \times 1$ column vector. From (7), the complete stiffness matrix of the manipulator in Cartesian space is obtained as

$$
K_{X}=J^{-T}\left(K_{\Theta}-K_{C}\right) J^{-1} .
$$

The force vector $F$ can be a dynamic force or a static force such as a payload carried by the manipulator. In this study, it is assumed that it is in the form of $F=\left[F_{x}, F_{y}, F_{z}\right]^{T}$. Such a force vector will generate a deflection of $\Delta X=[\delta x, \delta y, \delta z]^{T}$, which is a positioning error. If the joint stiffness $K_{\theta i}$ values are identified accurately using some experimental deflection and force data, the stiffness $K_{X}$ can subsequently be calculated from (7) for any external force vector without needing any other experimental data. By substituting (10) into (6) and solving for $\Delta X$ gives

$$
\Delta X=K_{X}^{-1} F=g\left(\Theta, K_{\theta i}, F\right) .
$$

For a given manipulator configuration where a force vector $F$ causes the deflection vector $\Delta X,(11)$ is nonlinear with unknown joint stiffness $K_{\theta i}$ values, which can be estimated using a least squares estimation algorithm. Since the stiffness of a joint is a local property, and the topology of a revolute joint does not change with the movement of the manipulator, it is assumed that the stiffness matrix $K_{\theta}$ is constant for revolute jointed manipulators.

\section{A. Nonlinear Least Square}

Based on the nonlinear deflection model $g(\cdots)$ of the manipulator expressed with (11), the joint stiffness values are estimated by minimizing the summed square of the error vector associated with $n$ number of measurements

$$
\mathrm{E}=\sum_{k=1}^{n}\left([\Delta D]_{k}\right)^{T}[\Delta D]_{k}
$$

where $\Delta D_{k}$ is the error vector given by

$$
\Delta D_{k}=\left(\Delta X_{r}-\Delta X_{m}\right)_{k} .
$$

$\Delta X_{r}$ is the measured (true) end point deflection vector under a range of payloads, and $\Delta X_{m}$ is the deflection vector calculated from (11) for the same payload. This is basically a nonlinear least square optimization problem that can be solved using either the interior-reflective Newton method or Levenburg-Marquardt algorithm. These are two efficient optimization algorithms for large-scale nonlinear problems. The former, which is based on the method of preconditioned conjugate gradients, can solve difficult nonlinear problems more efficiently than the latter [21]. In this study, both algorithms are implemented for the nonlinear least square estimation of the parameters. The solutions converged to the same numerical values. The procedure was realized iteratively until the deflection error was small enough to meet a termination condition of $10^{-15}$.

\section{B. Experimental Setup and Results}

The key elements of the experimental setup depicted in Fig. 2 are the laser tracker, retroreflector, the robot (Motoman SK120), and a fixture connected to the manipulator end point in order to exert forces (loads) along the three orthogonal axes of the manipulator end point. As shown in Fig. 3, a cable-pulley system was used to generate the needed force vector. The deadweights in the range of $0-50 \mathrm{~kg}$ with $10-\mathrm{kg}$ increments were suspended to the free end of the cable. The three resulting force components were measured via a wrist force/torque sensor. The laser-based dynamic measurement system was calibrated to measure the manipulator end point with respect to the manipulator base frame. The manipulator was commanded to 20 different well-spaced configurations within the manipulator workvolume, which had been determined heuristically to cover the range of motion of all the active joints of the 


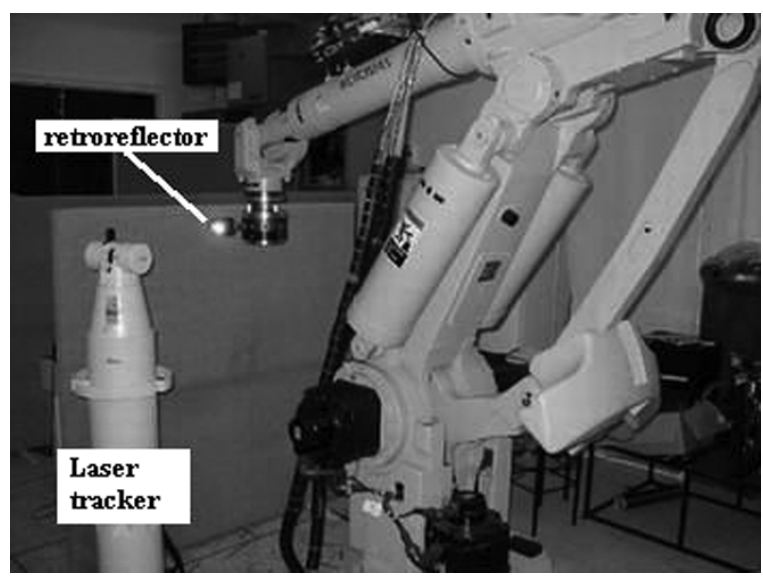

Fig. 2. Experimental setup.

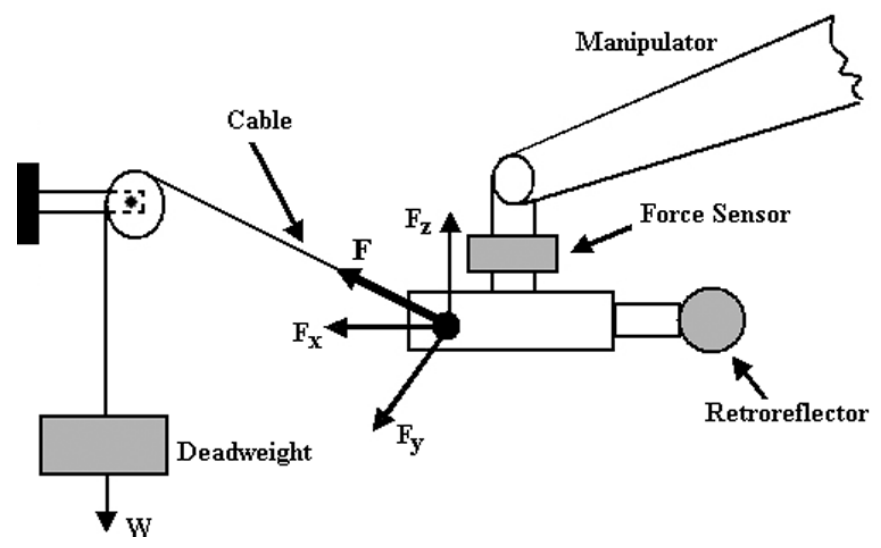

Fig. 3. Schematic of loading setup.

manipulator. The position of the manipulator joints responsible for the orientation were such that the manipulator end point was always perpendicular to the ground during the course of this research. The manipulator position controller is the one provided by the original equipment manufacturer. In order to minimize the effects of measurement noise, the PC-based data measurement and recording system of the laser tracker takes 100 measurements for the same configuration and provides the average of these measurements as the measurand. For each selected configuration, first of all, the Cartesian position of the manipulator end point without payload was recorded by the laser tracker, and followed by measurements of the Cartesian position of the manipulator for each level of payload, and finally the difference between the unloaded and loaded measurements were taken to obtain the deflection data for that particular force vector.

Some typical experimental force-deflection curves are depicted in Figs. 4-6. It must be noted that the Cartesian stiffness of the manipulator changes significantly from one configuration to the other.

The $3 \times 1$ error vector $\Delta D_{k}$ in (12) is obtained for each selected configuration, and the resulting $\Delta D_{k}$ is stacked at the end of the previous ones such that it becomes a vector of $(20 \times 3) \times$ 1 after 20 measurements. This procedure is repeated for each nonzero loading condition to test the linearity of the manipulator stiffness matrices $K_{X}$ and $K_{\Theta}$. A good initial guess helps a least
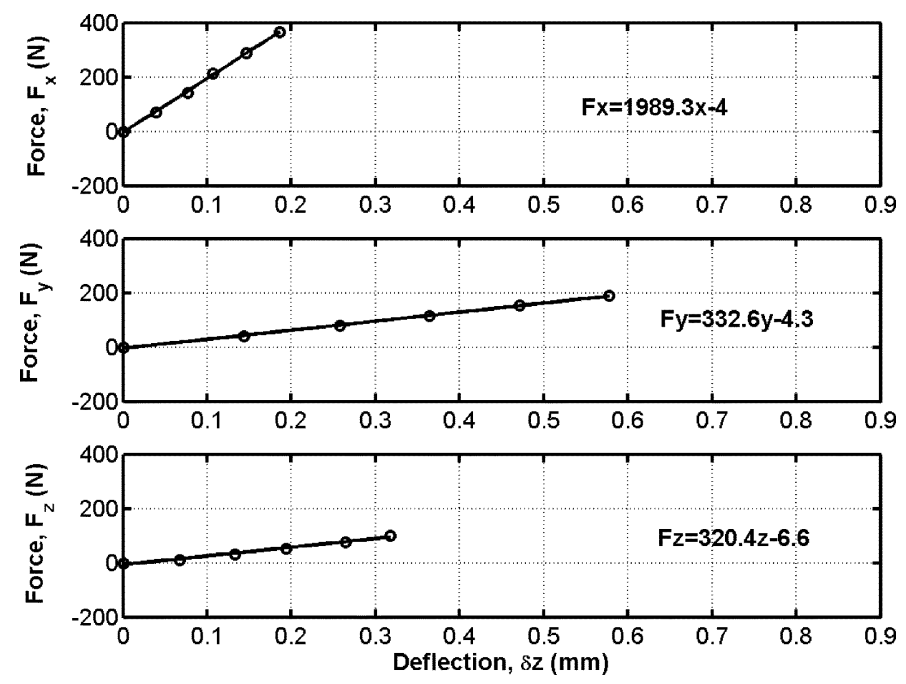

Fig. 4. Force-deflection curve for the configuration of $\theta_{1}=-89.3803^{\circ}$, $\theta_{2}=-62.9809^{\circ}, \theta_{3}=21.2489^{\circ}, \theta_{4}=0^{\circ}, \theta_{5}=-111.2492^{\circ}, \theta_{6}=$ $-90^{\circ}$.
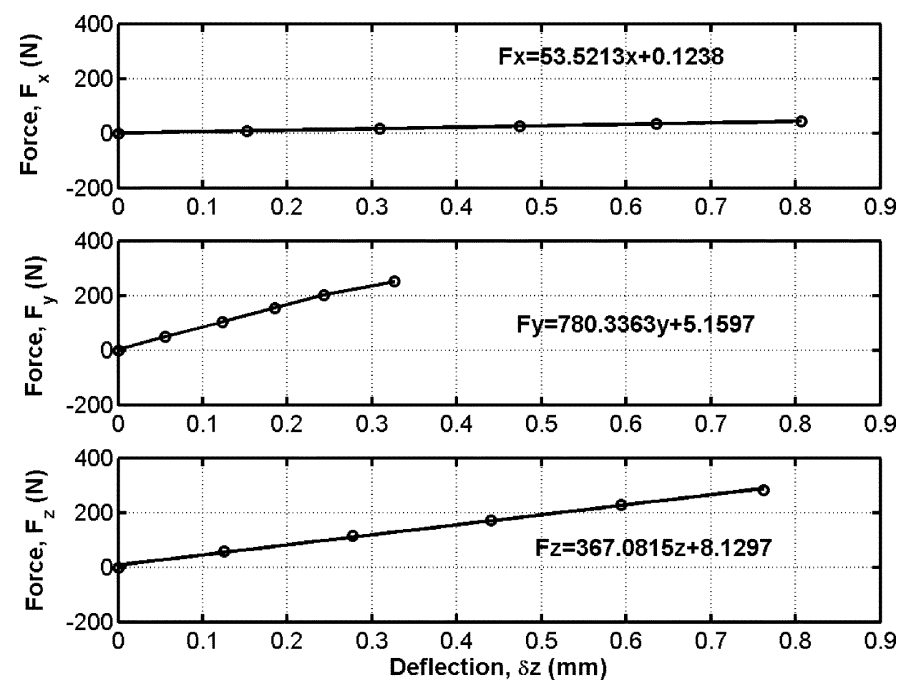

Fig. 5. Force-deflection curve for the configuration of $\theta_{1}=98.1065^{\circ}, \theta_{2}=$ $23.2088^{\circ}, \theta_{3}=-20.7957^{\circ}, \theta_{4}=0^{\circ}, \theta_{5}=-69.2041^{\circ}, \theta_{6}=90^{\circ}$.

square estimation algorithm to converge quickly without experiencing any numerical singularities. Typical stiffness values of $K_{\Theta}=$ diag. $\left[10^{8}, 10^{8}, 10^{8}\right] \mathrm{N} . \mathrm{mm} / \mathrm{rad}$ are chosen as the initial values. The estimated values of the joint stiffness matrix are found to be $K_{\Theta}=\operatorname{diag} \cdot\left[1.4098 \times 10^{9}, 4.0076 \times 10^{8}, 9.3528 \times\right.$ $\left.10^{8}\right] \mathrm{N} . \mathrm{mm} / \mathrm{rad}$, with a residual norm of 2.2106 described by (12). Different initial values of $K_{\Theta}$ led to the same joint stiffness values, which are the global solutions. This supports our assumption that the joint stiffness values are constant and do not change with the manipulator configuration. The identified stiffness values have been utilized in evaluating the maximum and minimum limits of the manipulator stiffness for robotic fiber placement [22].

\section{Stiffness Verification}

For three manipulator configurations where a number of external force vectors act at the manipulator end point, the Cartesian stiffness matrix is calculated from (10) and subsequently is 
TABLE II

STIFFNESS VERIFICATION RESULTS FOR THREE MANIPULATOR CONFIGURATIONS

\begin{tabular}{|c|c|c|c|}
\hline $\begin{array}{l}\text { Configurations } \\
\left(\theta_{1} \ldots \theta_{6}\right)(\text { Degrees })\end{array}$ & $\begin{array}{c}\text { Force Vector } \\
\left(\mathrm{F}_{\mathrm{x}}, \mathrm{F}_{\mathrm{y}}, \mathrm{F}_{\mathrm{z}}\right) \text { (Newton) }\end{array}$ & $\begin{array}{l}\text { Deflection Calculated } \\
(\delta \mathrm{x}, \delta \mathrm{y}, \delta \mathrm{z})(\mathrm{mm})\end{array}$ & $\begin{array}{l}\text { Deflection Measured } \\
(\delta \mathrm{x}, \delta \mathrm{y}, \delta \mathrm{z})(\mathrm{mm})\end{array}$ \\
\hline \multirow{5}{*}{$\begin{array}{l}-33.2004,15.9,- \\
55.5957,0,- \\
34.4045,-90.0\end{array}$} & $9.2295,72.9220,44.1053$ & $0.0288,0.1600,0.0483$ & $0.0300,0.1750,0.0560$ \\
\hline & $18.2141,145.0883,86.5843$ & $0.0583,0.3187,0.0944$ & $0.0550,0.3560,0.1090$ \\
\hline & $27.3052,217.9862,128.0153$ & $0.0886,0.4794,0.1389$ & $0.0800,0.5310,0.1490$ \\
\hline & $36.3531,290.6975,169.5228$ & $0.1187,0.6396,0.1834$ & $0.1050,0.7090,0.1970$ \\
\hline & $45.5240,364.5018,211.3439$ & $0.1494,0.8023,0.2282$ & $0.1430,0.8520,0.2480$ \\
\hline \multirow{5}{*}{$\begin{array}{c}32.5391,32.4702,- \\
19.0085, \quad 0,- \\
70.9913,-90.0\end{array}$} & $-5.3855,-73.2743,-26.0841$ & $-0.0606,-0.2311,-0.0464$ & $-0.0710,-0.2580,-0.0520$ \\
\hline & $-11.4628,-144.0103,-49.4234$ & $-0.1153,-0.4545,-0.0843$ & $730,-0.1030$ \\
\hline & $-17.7331,-216.8812,-72.7169$ & $-0.1711,-0.6850,-0.1208$ & $-0.2070,-0.7120,-0.1300$ \\
\hline & $-23.8670,-287.3281,-94.4728$ & $-0.2241,-0.9081,-0.1535$ & $-0.2307,-0.9540,-0.1680$ \\
\hline & $-30.9889,-367.4165,-118.9137$ & $-0.2837,-1.1618,-0.1895$ & $-0.3180,-1.1660,-0.2020$ \\
\hline \multirow{5}{*}{$\begin{array}{cc}60.9275, & 14.7064 \\
6.0766, & 0,- \\
96.0770, & 90.0\end{array}$} & $-37.8347,34.4328,20.5028$ & $-0.0970,0.0800,0.0148$ & $-0.0990,0.1100,0.0220$ \\
\hline & $-84.3213,76.8196,43.4368$ & $-0.2176,0.1813,0.0389$ & $-0.2220,0.2080,0.0510$ \\
\hline & $-115.1772,105.1296,57.8106$ & $-0.2983,0.2501,0.0573$ & $-0.3090,0.2810,0.0640$ \\
\hline & $-172.6610,158.1154,83.4755$ & $-0.4494,0.3803,0.0948$ & $-0.4850,0.4200,0.1002$ \\
\hline & $-196.4681,180.1925,94.1010$ & $-0.5119,0.4346,0.1105$ & $-0.5470,0.4830,0.1470$ \\
\hline
\end{tabular}
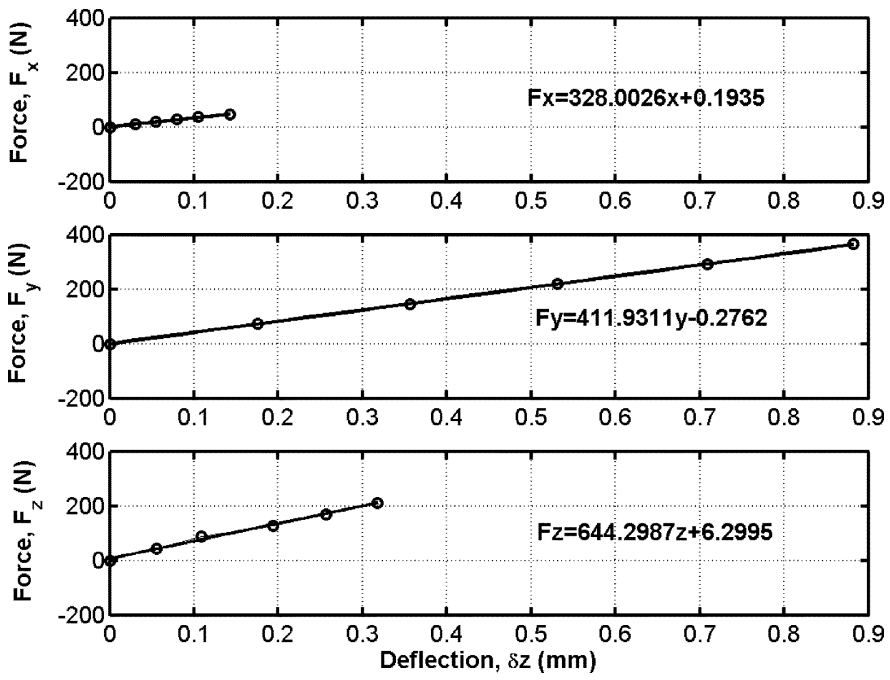

Fig. 6. Force-deflection curve for the configuration of $\theta_{1}=-13.6727^{\circ}$, $\theta_{2}=-10.9660^{\circ}, \theta_{3}=-37.4489^{\circ}, \theta_{4}=0^{\circ}, \theta_{5}=-52.5514^{\circ}, \theta_{6}=$ $-90^{\circ}$.

substituted into (6) to obtain the resulting deflection of the manipulator end point. These deflection data are compared to the deflection data obtained using the laser-based tracking system. These results are shown in Table II. It must be noted that the difference between the calculated and measured data is negligibly small. This follows that the model utilized for stiffness identification is valid and effective in estimating the manipulator end point deflection under an external force vector. It must be noted that the results given in Table II are to verify the estimated joint stiffness values, not to compare how accurately both methods of stiffness formulation predict the deflection of the manipulator end point under an external force vector. Of course, this criterion could have been used to demonstrate the enhancement of "enhanced stiffness formulation" over "conventional stiffness formulation". This requires that the accuracy and resolution of the measurement system must be ultrahigh in order to provide credible results in this respect.
To further verify the experimental results and demonstrate that the enhanced stiffness formulation is valid, the net work done in Cartesian space and joint space are calculated for a range of external forces acting at the manipulator end point for the same configuration of the manipulator. Based on the conservation of energy, the external work done by applied loads on an elastic system in equilibrium is equivalent to the increase in the internal strain (elastic) energy stored in the system [23]. The Taylor series expansion of the stored energy leads to the following expression of the net work done by an external force vector $F$ in Cartesian space

$$
\Delta \mathrm{W}_{x}=F^{T} \Delta X+\frac{1}{2} \Delta X^{T} K_{x} \Delta X .
$$

The corresponding work done in joint space can be obtained by substituting the force and motion relationships, i.e., (3) and (4), between the Cartesian and joint spaces into (14). $K_{X}$ is described by (10). These give the net work done in joint space as

$$
\Delta \mathrm{W}_{\theta}=\Gamma^{T} \Delta X+\frac{1}{2} \Delta \Theta^{T}\left(K_{\theta}-K_{C}\right) \Delta \Theta .
$$

For the conventional stiffness formulation, the complimentary stiffness matrix $K_{C}$ is zero. For a conservative system, the works done in Cartesian and joint spaces must be equivalent. With this in mind, the resulting works for enhanced and conventional stiffness formulations are calculated for a number of manipulator configurations and loading conditions. It has been found that while the works in Cartesian and joint spaces are equivalent for the enhanced formulation, ${ }^{1}$ they are different for the conventional formulation. The numerical results for the conventional formulation are depicted in Table III. It must be noted that depending on the configuration and the magnitude of loading, the works done in Cartesian and joint spaces are not equivalent. This obviously demonstrates that the conventional

\footnotetext{
${ }^{1}$ Their numerical values are the same as the Cartesian work given in the third column of Table III. For the sake of brevity, they are not provided.
} 
TABLE III

WORKS CALCULATED FOR CONVENTIONAL STIFFNESS FORMULATION

\begin{tabular}{|c|c|c|c|}
\hline $\begin{array}{l}\text { Configurations } \\
\left(\theta_{1} \ldots \theta_{6}\right)(\text { Degrees })\end{array}$ & $\begin{array}{r}\text { Force Vector } \\
\left(\mathrm{F}_{\mathrm{x}}, \mathrm{F}_{\mathrm{y}}, \mathrm{F}_{\mathrm{z}}\right) \text { (Newton) }\end{array}$ & $\begin{array}{c}\text { Work in Cartesian Space } \\
\text { (Joule) }\end{array}$ & $\begin{array}{c}\text { Work in Joint Space CF } \\
\text { (Joule) }\end{array}$ \\
\hline \multirow{5}{*}{$\begin{array}{c}33.7176,25.8817, \\
-68.9311,0,-21.0686, \\
-90\end{array}$} & $70.3916,41.5479,10.8274$ & 15.9276 & 15.9271 \\
\hline & $141.6634,77.7620,31.9407$ & 57.2124 & 57.2094 \\
\hline & $214.4720,114.8795,53.6507$ & 120.0116 & 120.0024 \\
\hline & $289.3197,152.8731,76.6840$ & 212.0687 & 212.0477 \\
\hline & $364.3636,190.3636,100.1458$ & 324.9662 & 324.9274 \\
\hline \multirow{5}{*}{$\begin{array}{c}30.1395,44.5181, \\
5.4038,0,-95.4035,- \\
90\end{array}$} & $29.8945,60.1003,19.6415$ & 59.1960 & 59.1913 \\
\hline & $74.5583,119.8279,35.1475$ & 195.6889 & 195.6563 \\
\hline & $125.3127,187.6431,52.6577$ & 451.7432 & 451.6235 \\
\hline & $169.8202,247.1810,67.6436$ & 742.9267 & 742.6689 \\
\hline & $220.4150,314.7162,84.7484$ & 1211.5326 & 1210.9932 \\
\hline \multirow{5}{*}{$\begin{array}{c}91.0686,29.4383, \\
12.7021,0,-102.7020, \\
90\end{array}$} & $45.8939,41.9129,35.8635$ & 38.8084 & 38.8058 \\
\hline & $91.1697,83.1856,78.8780$ & 153.2174 & 153.1956 \\
\hline & $135.2980,163.3854,162.3367$ & 342.2626 & 342.1889 \\
\hline & $179.7123,163.3854,162.3367$ & 616.1872 & 616.0085 \\
\hline & $222.1806,201.5717,202.2606$ & 960.9724 & 960.6230 \\
\hline \multirow{5}{*}{$\begin{array}{c}-167.4226,-47.8021 \\
-20.7957,0,-69.2041 \\
90\end{array}$} & $52.7723,34.8188,50.1333$ & 61.0133 & 61.0145 \\
\hline & $104.8373,69.1931,99.4512$ & 259.6676 & 259.6797 \\
\hline & $158.7785,104.5551,150.6586$ & 610.4935 & 610.5386 \\
\hline & $209.5063,137.6227,197.8345$ & 1059.9549 & 1060.0593 \\
\hline & $263.4541,172.6293,248.0168$ & 1686.5746 & 1686.7834 \\
\hline \multirow{5}{*}{$\begin{array}{c}168.6301,19.8121 \\
-81.7685,0,-8.2312 \\
90\end{array}$} & $21.0535,57.3717,43.2363$ & 41.0334 & 41.0329 \\
\hline & $41.4312,112.5676,85.0804$ & 150.6607 & 150.6564 \\
\hline & $61.9385,167.9531,125.8612$ & 332.8650 & 332.8500 \\
\hline & $82.4825,223.0195,166.1294$ & 591.6924 & 591.6545 \\
\hline & $102.0751,275.1562,203.9803$ & 938.0831 & 938.0118 \\
\hline
\end{tabular}

stiffness formulation violates the conservation of energy. However, when the Cartesian stiffness matrix is calculated from (8) and substituted into (14), the net works in Cartesian and joint spaces are found to be equivalent. This can be possible only when either there is no force acting on the manipulator or the Jacobian matrix does not vary with the manipulator configuration.

Please note that the same experimental deflection and force data are used in calculating the works in Cartesian and joint spaces using enhanced and conventional formulations. While the works (Cartesian and joint space) calculated using enhanced stiffness formulation are precisely equal to each other, the same works calculated using 'conventional stiffness modeling' are different. It does not matter how small the difference is. The difference between the Cartesian and joint space works is due to the formulation, not due to the accuracy/resolution of the measurement systems used. The difference arises from the complementary stiffness matrix $K_{C}$. Obviously, while the enhanced stiffness modeling satisfies the principle of conservation of energy, the conventional stiffness does not. This demonstrates that the conventional stiffness formulation is incomplete and is valid under certain assumptions.

\section{STIFFNESS CHARACTERIZATION}

It has been reported [7], [11] that a conservative stiffness matrix in the linear Euclidean $R^{3 \times 3}$ space must (i) be symmetric, and (ii) satisfy the complete differential condition. What we call the complementary stiffness matrix in this study satisfies these two criteria-it is a conservative matrix. However, depending on the configuration of the manipulator and the external force, its positive definiteness cannot be guaranteed. In this section, we present a thorough investigation into the properties of the complementary stiffness matrix $K_{C}$ in order to evaluate under what conditions the difference matrix $K_{\theta}-K_{C}$ is positive definite or not. $K_{\theta}$ is always positive definite as it is a diagonal matrix with positive elements. As presented in Appendix II, the positive definiteness of $K_{\theta}-K_{C}$ determines that of the Cartesian stiffness matrix $K_{X}$ of the manipulator because of a congruence transformation between $K_{\theta}-K_{C}$ and $K_{X}$.

There are a number of ways to test the definiteness of a symmetric matrix [19]. We adopt the one based on the quadratic form of a matrix to search the definiteness of the difference matrix $K_{\theta}-K_{C}$. If the difference matrix is not positive definite (PD), we mean that it is negative definite (ND), negative semi-definite (NSD), positive semi-definite (PSD), or indefinite (ID). In such a case, the resulting Cartesian stiffness matrix $K_{X}$ is not positive definite, and it leads to an unstable contact or grip depending on what task the robot manipulator is performing [24], [25].

Corollary 1: If $K_{C}$ is negative definite or negative semi-definite, $K_{\theta}-K_{C}$ will always be positive definite. From definition of positive definiteness, $K_{\theta}-K_{C}$ is always $x^{T} K_{\theta} x-x^{T} K_{C} x>$ 0 for an arbitrary nonzero vector $x \in R^{m}$.

Corollary 2: If $K_{C}$ is positive definite, positive semi-definite, and indefinite, the positive definiteness of $K_{\theta}-K_{C}$ will depend on the relative values of corresponding elements of $K_{\theta}$ and $K_{C}$. The $K_{\theta}-K_{C}$ can be positive definite or not.

Assume that both $K_{\theta}$ and $K_{C}$ are $3 \times 3$ matrices given by

$$
\left[\begin{array}{ccc}
K_{1} & 0 & 0 \\
0 & K_{2} & 0 \\
0 & 0 & K_{3}
\end{array}\right] \text { and }\left[\begin{array}{lll}
C_{11} & C_{12} & C_{13} \\
C_{21} & C_{22} & C_{23} \\
C_{31} & C_{32} & C_{33}
\end{array}\right] .
$$


TABLE IV

RESUlts of Definiteness Test FOR MATRIX $K_{C}$ FOR EIGHT DifFERENT LOAding CASES

\begin{tabular}{c|c|c|c|c|r}
\hline Case & PD & PSD & ND & NSD & ID \\
\hline 1 & 3047 & 0 & 717 & 0 & 2060 \\
\hline 2 & 2785 & 0 & 360 & 0 & 2679 \\
\hline 3 & 3063 & 0 & 422 & 0 & 2339 \\
\hline 4 & 3041 & 0 & 706 & 0 & 2077 \\
\hline 5 & 2777 & 0 & 361 & 0 & 2686 \\
\hline 6 & 2791 & 0 & 362 & 0 & 2671 \\
\hline 7 & 2791 & 0 & 362 & 0 & 2671 \\
\hline 8 & 3047 & 0 & 717 & 0 & 2060 \\
\hline
\end{tabular}

For a positive definite $K_{\theta}-K_{C}$, the following three conditions (the determinants of the primary minors) have to be satisfied simultaneously as follows.

i) $K_{1}-C_{11}>0.0$.

ii) $\left(K_{1}-C_{11}\right)\left(K_{2}-C_{22}\right)-C_{12}^{2}>0.0$.

iii) $\left\{\begin{array}{l}\left(K_{1}-C_{11}\right)\left[\left(K_{2}-C_{22}\right)\left(K_{3}-C_{33}\right)-C_{23}^{2}\right] \\ -C_{12}\left[C_{31} C_{23}+C_{12}\left(K_{3}-C_{33}\right)\right] \\ -C_{13}\left[C_{21} C_{32}+C_{31}\left(K_{2}-C_{22}\right)\right]\end{array}\right\}>$

If any of these conditions is not satisfied, $K_{\theta}-K_{C}$ will not be positive definite. This method of testing the definiteness of a matrix is deliberately followed to quantify the order of the external forces needed to generate a nonpositive definite $K_{\theta}-$ $K_{C}$, as numerically demonstrated in Section VI.

\section{Computational Analysis}

The definiteness of the matrix $K_{C}$ depends on the manipulator configuration and the magnitude and direction of the external force vector. For the identified joint stiffness matrix $K_{\Theta}=$ diag. $\left[1.4098 \times 10^{9}, 4.0076 \times 10^{8}, 9.3528 \times\right.$ $\left.10^{8}\right] \mathrm{N} . \mathrm{mm} / \mathrm{rad}$, and a range of external forces acting on the manipulator, the definiteness of $K_{C}$ is evaluated numerically. The manipulator has the motion ranges of $-180^{\circ} \leq \theta_{1} \leq 180^{\circ}$, $-70^{\circ} \leq \theta_{2} \leq 70^{\circ}$, and $-115^{\circ} \leq \theta_{3} \leq 35^{\circ}$ for its first three joints. These motion ranges are implemented with a step size of 0.2 radian or $11.46^{\circ}$. It is assumed that the magnitude of each component of the force vector is 100 Newton with the following directions.

1) All of the components $F_{x}, F_{y}, F_{z}$ are positive.

2) All of the components $F_{x}, F_{y}, F_{z}$ are negative.

3) $F_{x}$ is negative, $F_{y}$ and $F_{z}$ are positive.

4) $F_{y}$ is negative, $F_{x}$ and $F_{z}$ are positive.

5) $F_{z}$ is negative, $F_{x}$ and $F_{y}$ are positive.

6) $F_{x}$ is positive, $F_{y}$ and $F_{z}$ are negative.

7) $F_{y}$ is positive, $F_{x}$ and $F_{z}$ are negative.

8) $F_{z}$ is positive, $F_{x}$ and $F_{y}$ are negative.

For the specified conditions and numerical values, the definiteness of $K_{C}$ and $K_{\theta}-K_{C}$ are determined. The definiteness test results for $K_{C}$ are shown in Table IV in terms of the number of positive definite (PD) and nonpositive definite configurations for the eight loading conditions (force application directions) outlined above. It must be noted that $K_{C}$ is not

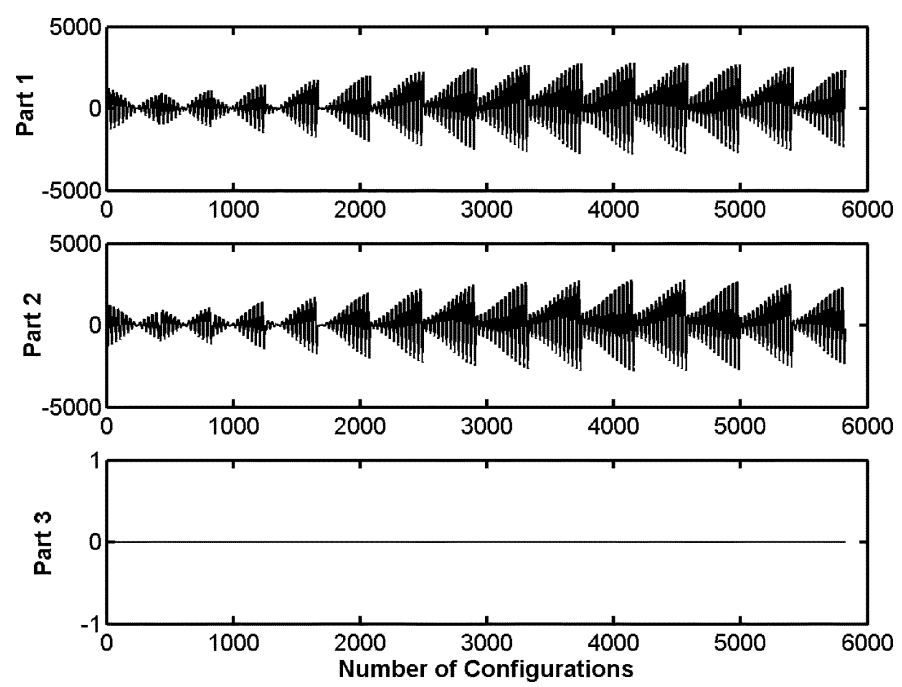

Fig. 7. Variation of the partial derivatives forming $C_{11}$.

always positive definite. However, it has been found that the difference matrix $K_{\theta}-K_{C}$ is always positive definite and so is $K_{X}$ for the manipulator considered in this study. This follows that the energy stored in the system can be released in a stable manner.

It must be noted from Corollary 2 that when the nonnegative magnitudes of the diagonal elements $\left(C_{11}, C_{22}, C_{33}\right)$ of $K_{C}$ become larger than the corresponding elements of $K_{\theta}$, it is obvious that $K_{\theta}-K_{C}$ can no longer be positive definite, and neither can $K_{X}$. In order to evaluate the limits of the external forces leading to such a scenario, the components of the partial derivatives forming the diagonal elements are calculated for the ranges of motion specified above and are shown in Figs. 7-9 for the diagonal elements $C_{11}, C_{22}$ and $C_{33}$, respectively. It must be noted that the top, middle and bottom plots in these figures correspond to the "Part 1," "Part 2," and "Part 3" of the right-hand side of (16), respectively. This follows that the sum of these parts represents the magnitude of the diagonal elements $\left(C_{11}, C_{22}\right.$, $C_{33}$ ) of $K_{C}$ for a unit force vector

$$
\begin{aligned}
& C_{\mathrm{ii}}=\underbrace{\frac{\partial J_{k 1}}{\partial \theta_{k}}}_{\text {Part } 1} F_{x}+\underbrace{\frac{\partial J_{k 2}}{\partial \theta_{k}}}_{\text {Part } 2} F_{y}+\underbrace{\frac{\partial J_{k 3}}{\partial \theta_{k}}}_{\text {Part } 3} F_{z}, \\
& \text { for } k=1,2,3 \text { and } i=1,2,3 \text {. }
\end{aligned}
$$



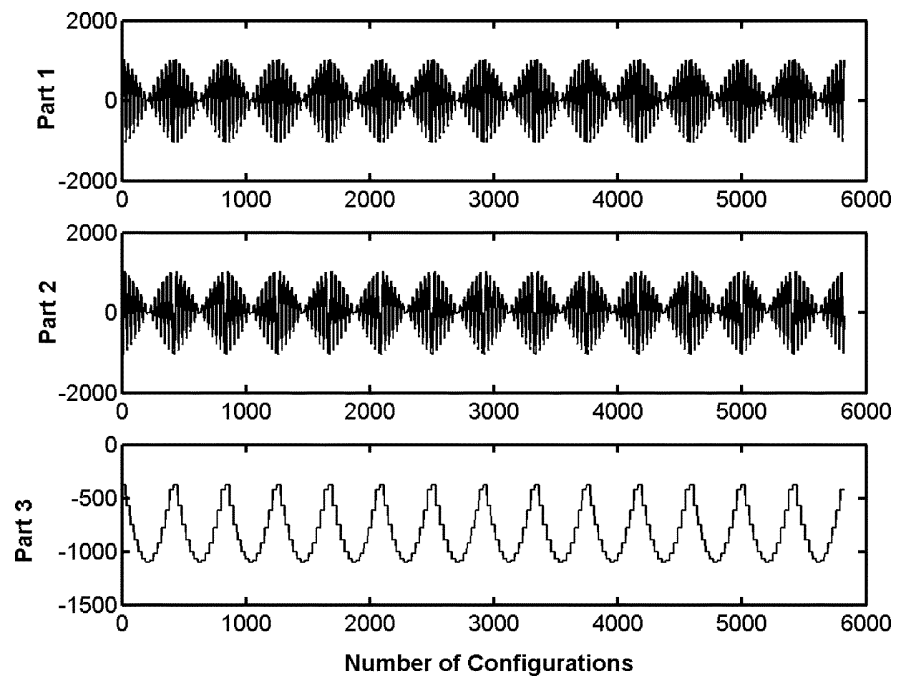

Fig. 8. Variation of the partial derivatives forming $C_{22}$.
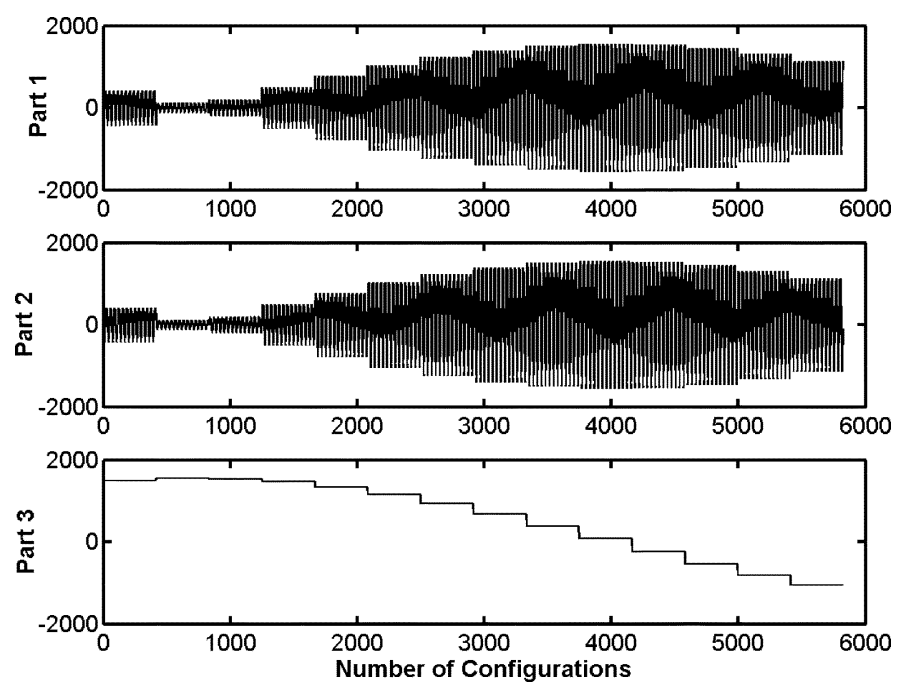

Fig. 9. Variation of the partial derivatives forming $C_{33}$.

With reference to Figs. 7-9 and (16), the forces as large as $10 \mathrm{kN}$ are needed in order to reach and pass the order of the joint stiffness values identified in Section IV-B. Hence, the constraints given in Corollary 2 for a positive definite $K_{\theta}-K_{C}$ will be violated. No robot manipulator can withstand such a force level. However, it can be argued that an elastic-jointed manipulator will most probably have a nonpositive definite Cartesian stiffness matrix.

\section{CONCLUSION}

We have presented the results and implications of our study into the enhanced stiffness modeling, identification, and characterization of robot manipulators based on the experimental data provided by a wrist $\mathrm{F} / \mathrm{T}$ sensor and a high precision
Cartesian position sensor (laser tracker). The estimated joint stiffness matrix is verified through a set of experimental deflection data. Also, the net work done in Cartesian and joint spaces by an external force vector are computed for enhanced and conventional stiffness formulations. It has been proven that the conventional stiffness formulation does not obey the principle of conservation of energy. This further demonstrates that the conventional stiffness formulation is incomplete. Based on the estimated joint stiffness constants, the limits and conditions of the positive definiteness (stability) of the Cartesian stiffness matrix are also evaluated. It has been shown that although the component of the stiffness matrix differentiating the enhanced stiffness model from the conventional one is not always positive definite, the resulting stiffness matrix can still be positive definite. The principal contribution of this study is that new experimental results supporting the enhanced stiffness formulation through stiffness identification, verification, and characterization.

\section{APPENDIX I}

With reference to (3), the $3 \times 3$ Jacobian matrix of the manipulator is derived as

$$
\left[\begin{array}{lll}
J_{11} & J_{12} & J_{13} \\
J_{21} & J_{22} & J_{23} \\
J_{31} & J_{32} & J_{33}
\end{array}\right]
$$

where

$$
\begin{aligned}
J_{11}= & L_{4} \cos \theta_{1} \sin \theta_{4}-D_{3} \cos \theta_{1}-L_{1} \sin \theta_{1} \\
& +L_{6} \cos \theta_{1} \sin \theta_{4} \cos \theta_{5} \cos \theta_{6}+D_{5} \cos \theta_{1} \cos \theta_{4} \\
& +L_{6} \cos \theta_{1} \cos \theta_{4} \sin \theta_{6}+L_{5} \cos \theta_{1} \sin \theta_{4} \cos \theta_{5} \\
& +D_{6} \cos \theta_{1} \sin \theta_{4} \sin \theta_{5}-D_{6} \sin \theta_{1} \cos \theta_{3} \cos \theta_{5} \\
& +L_{5} \sin \theta_{1} \cos \theta_{3} \sin \theta_{5}-L_{5} \sin \theta_{1} \sin \theta_{3} \cos \theta_{4} \cos \theta_{5} \\
& +L_{6} \sin \theta_{1} \cos \theta_{3} \sin \theta_{5} \cos \theta_{6}-L_{3} \sin \theta_{1} \sin \theta_{3} \\
& -L_{6} \sin \theta_{1} \sin \theta_{3} \cos \theta_{4} \cos \theta_{5} \cos \theta_{6} \\
& +L_{6} \sin \theta_{1} \sin \theta_{3} \sin \theta_{4} \sin \theta_{6} \\
& -D_{6} \sin \theta_{1} \sin \theta_{3} \cos \theta_{4} \sin \theta_{5} \\
& +D_{5} \sin \theta_{1} \sin \theta_{3} \sin \theta_{4}-L_{4} \sin \theta_{1} \sin \theta_{3} \cos \theta_{4} \\
& -D_{4} \sin \theta_{1} \cos \theta_{3}-L_{2} \sin \theta_{1} \sin \theta_{3} \sin \left(\theta_{2}-\theta_{3}\right) \\
& \left.+L_{2} \sin \theta_{1} \cos \theta_{3} \cos \theta_{2}-\theta_{3}\right) \\
J_{12}= & L_{2} \cos \theta_{1} \sin \theta_{2} \\
J_{13}= & L_{6} \cos \theta_{1} \sin \theta_{3} \sin \theta_{5} \cos \theta_{6} \\
& -L_{6} \cos \theta_{1} \cos \theta_{3} \sin \theta_{4} \sin \theta_{6}-D_{4} \cos \theta_{1} \sin \theta_{3} \\
& +L_{3} \cos \theta_{1} \cos \theta_{3}+D_{6} \cos \theta_{1} \cos \theta_{3} \cos \theta_{4} \sin \theta_{5} \\
& +L_{5} \cos \theta_{1} \cos \theta_{3} \cos \theta_{4} \cos \theta_{5} \\
& +L_{6} \cos \theta_{1} \cos \theta_{3} \cos \theta_{4} \cos \theta_{5} \cos \theta_{6} \\
& +L_{4} \cos \theta_{1} \cos \theta_{3} \cos \theta_{4}-D_{6} \cos \theta_{1} \sin \theta_{3} \cos \theta_{5} \\
& -D_{5} \cos \theta_{1} \cos \theta_{3} \sin \theta_{4}+L_{5} \cos \theta_{1} \sin \theta_{3} \sin \theta_{5} \\
&
\end{aligned}
$$




$$
\begin{aligned}
& J_{21}=-D_{4} \cos \theta_{1} \cos \theta_{3}+L_{3} \cos \theta_{1} \sin \theta_{3} \\
& \text { - } L_{6} \cos \theta_{1} \cos \theta_{3} \sin \theta_{5} \cos \theta_{6} \\
& +D_{6} \cos \theta_{1} \sin \theta_{3} \cos \theta_{4} \sin \theta_{5} \\
& +D_{6} \cos \theta_{1} \cos \theta_{3} \cos \theta_{5}-D_{5} \cos \theta_{1} \sin \theta_{3} \sin \theta_{4} \\
& +L_{6} \sin \theta_{1} \sin \theta_{4} \cos \theta_{5} \cos \theta_{6} \\
& +D_{6} \sin \theta_{1} \sin \theta_{4} \sin \theta_{5}+L_{6} \sin \theta_{1} \cos \theta_{4} \sin \theta_{6} \\
& +L_{5} \sin \theta_{1} \sin \theta_{4} \cos \theta_{5}+L_{4} \sin \theta_{1} \sin \theta_{4} \\
& +D_{5} \sin \theta_{1} \cos \theta_{4}-D_{1} \sin \theta_{1} \\
& +L_{6} \cos \theta_{1} \sin \theta_{3} \cos \theta_{4} \cos \theta_{5} \cos \theta_{6} \\
& \text { - } L_{6} \cos \theta_{1} \sin \theta_{3} \sin \theta_{4} \sin \theta_{6} \\
& +L_{4} \cos \theta_{1} \sin \theta_{3} \cos \theta_{4}-L_{5} \cos \theta_{1} \cos \theta_{3} \sin \theta_{5} \\
& +L_{5} \cos \theta_{1} \sin \theta_{3} \cos \theta_{4} \cos \theta_{5}+L_{1} \cos \theta_{1} \\
& \text { - } L_{2} \cos \theta_{1} \cos \theta_{3} \cos \left(\theta_{2}-\theta_{3}\right) \\
& +L_{2} \cos \theta_{1} \sin \theta_{3} \sin \left(\theta_{2}-\theta_{3}\right) \\
& J_{22}=L_{2} \sin \theta_{1} \sin \theta_{2} \\
& J_{23}=-D_{5} \sin \theta_{1} \cos \theta_{3} \sin \theta_{4}+L_{5} \sin \theta_{1} \cos \theta_{3} \cos \theta_{4} \cos \theta_{5} \\
& +L_{3} \sin \theta_{1} \cos \theta_{3}-L_{6} \sin \theta_{1} \cos \theta_{3} \sin \theta_{4} \sin \theta_{6} \\
& +L_{6} \sin \theta_{1} \sin \theta_{3} \sin \theta_{5} \cos \theta_{6}+L_{4} \sin \theta_{1} \cos \theta_{3} \cos \theta_{4} \\
& -D_{4} \sin \theta_{1} \sin \theta_{3}+L_{5} \sin \theta_{1} \sin \theta_{3} \sin \theta_{5} \\
& +D_{6} \sin \theta_{1} \cos \theta_{3} \cos \theta_{4} \sin \theta_{5}-D_{6} \sin \theta_{1} \sin \theta_{3} \cos \theta_{5} \\
& +L_{6} \sin \theta_{1} \cos \theta_{3} \cos \theta_{4} \cos \theta_{5} \cos \theta_{6} \\
& J_{31}=0 \\
& J_{32}=L_{2} \cos \theta_{2} \\
& J_{33}=D_{5} \sin \theta_{3} \sin \theta_{4}-L_{4} \sin \theta_{3} \cos \theta_{4} \\
& -L_{5} \sin \theta_{3} \cos \theta_{4} \cos \theta_{5}-D_{4} \cos \theta_{3} \\
& +L_{6} \sin \theta_{3} \sin \theta_{4} \sin \theta_{6}-D_{6} \sin \theta_{3} \cos \theta_{4} \sin \theta_{5} \\
& \text { - } L_{6} \sin \theta_{3} \cos \theta_{4} \cos \theta_{5} \cos \theta_{6}-D_{6} \cos \theta_{3} \cos \theta_{5} \\
& +L_{5} \cos \theta_{3} \sin \theta_{5}-L_{3} \sin \theta_{3}+L_{6} \cos \theta_{3} \sin \theta_{5} \cos \theta_{6} \text {. }
\end{aligned}
$$

\section{APPENDIX II}

Consider pre- and post-multiplying both sides of (10) by a nonzero arbitrary vector $z^{T} \in R^{m}$ and $z \in R^{m}$, respectively

$$
\begin{aligned}
z^{T}\left(K_{X}\right) z & =z^{T} J^{-T}\left(K_{\Theta}-K_{C}\right) J^{-1} z \\
& =\left(J^{-1} z\right)^{T}\left(K_{\Theta}-K_{C}\right) J^{-1} z \\
& =\bar{z}^{T}\left(K_{\Theta}-K_{C}\right) \bar{z}
\end{aligned}
$$

where $\bar{z}=J^{-1} z$, a linear change of variables. If $K_{X}$ is positive definite, $z^{T} K_{X} z>0$ and so is $\bar{z}^{T}\left(K_{\Theta}-K_{C}\right) \bar{z}>0$. This requires that the difference matrix $K_{\theta}-K_{C}$ is also positive definite. Clearly, the definiteness of $K_{\theta}-K_{C}$ determines that of the Cartesian stiffness matrix $K_{X}$, and vice versa.

Alternatively, the positive definiteness of $K_{\theta}-K_{C}$ can be characterized by the difference of two quadratic forms. As $K_{\theta}$ is a diagonal matrix with positive elements on the main diagonal, its quadratic form is already in a canonical form. If the quadratic form of the matrix $K_{C}$ is brought to a canonical form with a linear change of variables $x=P y$, the definiteness of $K_{\theta}-K_{C}$ can be characterized with the difference of two canonical forms. This follows that

$$
x^{T} K_{C} x=(P y)^{T} K_{C}(P y) \Rightarrow y^{T}\left(P^{T} K_{C} P\right) y .
$$

It is needed to determine the matrix $P$ such that $\left(P^{T} K_{C} P\right)$ is diagonal, and hence the quadratic form from (19) will be canonical with new variable $y$. As it is well known [19], if the columns of $P$ are the normalized eigenvectors of $K_{C},\left(P^{T} K_{C} P\right)$ will be a diagonal matrix such that its diagonal elements are the corresponding eigenvalues of $K_{C}$. Hence, the definiteness of the two canonical forms can be determined from

$$
\begin{aligned}
f\left(x, y, \lambda_{i}^{\theta}, \lambda_{i}^{C}\right)=\left(\lambda_{1}^{\theta} x_{1}^{2}+\right. & \left.\lambda_{2}^{\theta} x_{2}^{2}+\lambda_{1}^{\theta} x_{3}^{2}\right) \\
& -\left(\lambda_{1}^{C} y_{1}^{2}+\lambda_{2}^{C} y_{2}^{2}+\lambda_{1}^{C} y_{3}^{2}\right)
\end{aligned}
$$

where $\lambda_{i}^{\theta}$ and $\lambda_{i}^{C}$ are the eigenvalues of $K_{\theta}$ and $K_{C}$, respectively. The reverse transformation can be accomplished from $y=P^{-1} x$. The numerical value obtained from (20) using the eigenvalues of $K_{\theta}$ and $K_{C}$ will indicate whether the difference matrix is positive definite or not.

\section{REFERENCES}

[1] G. Alici and R. W. Daniel, "Static friction effects during hard-on-hard contact tasks and their implications for manipulator design," Int. J. Robot. Res., vol. 13, no. 6, pp. 508-520, 1994.

[2] — - "Robotic drilling under force control: execution of a task," in Proc. 1994 IEEE/RSJ Int. Conf. Intelligent Robots and Systems, Münich, Germany, 1994, pp. 1618-1625.

[3] H. Bruyninckx and J. D. Schutter, "Specification of force-controlled actions in the 'task frame formalism'-a synthesis," IEEE Trans. Robot. Autom., vol. 12, no. 4, pp. 581-589, Aug. 1996.

[4] M. T. Mason and J. K. Salisbury, Robot Hands and the Mechanics of Manipulation. Cambridge, MA: MIT Press, 1985.

[5] H. R. Choi, W. K. Chung, and Y. Youm, "Stiffness analysis and control of redundant manipulators," in Proc. IEEE Int. Conf. Robotics and Automation, 1994, pp. 689-695.

[6] B. J. Li and R. A. Freeman, "Synthesis of actively adjustable springs by antagonistic redundant actuation," ASME J. Dyn Syst., Measur. Contr., vol. 114, pp. 454-461, 1992.

[7] S. F. Chen and I. Kao, "Conservative congruence transformation for joint and Cartesian stiffness matrices of robotic hands and fingers," Int. J. Robot. Res., vol. 19, no. 9, pp. 835-847, 2000.

[8] I. Kao and C. Ngo, "Properties of grasp stiffness matrix and conservative control strategy," Int. J. Robot. Res., vol. 18, no. 2, pp. 159-167, 1999.

[9] Y. Li and I. Kao, "A review of modeling of soft-contact fingers and stiffness control for dextrous manipulation in robotics," in Proc. IEEE Int Conf. Robot. Autom., Seoul, Korea, 2001, pp. 3055-3060.

[10] Y. Li, S. F. Chen, and I. Kao, "Stiffness control and transformation for robotic systems with coordinate and noncoordinate bases," in Proc. IEEE Int. Conf. Robot. Autom., Washington, DC, 2002, pp. 550-555.

[11] M. H. Ang and G. B. Andeen, "Specifying and achieving passive compliance based on manipulator structure," IEEE Trans. Robot. Autom., vol. 11, no. 4, pp. 504-515, Aug. 1995.

[12] M. H. Ang, W. Wang, R. N. K. Loh, and T. S. Low, "Passive compliance from robot limbs and its usefulness in robotic automation," J. Intell. Robot. Syst., vol. 20, pp. 1-21, Sep. 1997.

[13] I. Kao, M. R. Cutkosky, and R. S. Johansson, "Robotic stiffness control and calibration as applied to human grasping tasks," IEEE Trans. Robot. Autom., vol. 13, no. 4, pp. 557-566, Aug. 1997.

[14] S. Huang and J. M. Schimmels, "The bounds and realization of spatial stiffnesses achieved with simple springs connected in parallel," IEEE Trans. Robot. Autom., vol. 14, no. 3, pp. 466-475, Jun. 1998.

[15] M. Griffis and J. Duffy, "Global stiffness modeling of a class of simple compliant couplings," Mech. Mach. Theory, vol. 28, no. 2, pp. 207-224, 1993. 
[16] N. Ciblak and H. Lipkin, "Asymmetric Cartesian stiffness for the modeling of compliant robotic systems," in Proc. ASME Conf. Robotics: Kinematics, Dynamics, and Control, vol. 72, 1994, pp. 197-204.

[17] - "Synthesis of Cartesian stiffness for robotic applications," in Proc. IEEE Int. Conf. Robotics Automation, Detroit, MI, 1999, pp. $2147-2152$.

[18] G. Alici and B. Shirinzadeh, "Laser interferometry based robot kinematic error modeling and compensation," in Proc. IEEE/RSJ Int. Conf. Intelligent Robots and Systems, Las Vegas, NM, Oct. 2003, pp. 3588-3593.

[19] G. Strang, Linear Algebra and Its Applications, 2nd ed. Orlando, FL: Harcourt Brace Jovanovich, 1980, pp. 249-259.

[20] G. Alici and B. Shirinzadeh, "Exact stiffness analysis and mapping for a 3 -SPS +S parallel manipulator," in 7th Int. Conf. Automation Technologies, Chia-Yi, Taiwan, R.O.C., Sep. 2003, pp. 699-704.

[21] T. F. Coleman and Y. Li, "An interior trust Region approach for nonlinear minimization subject to bounds," J. Optim., vol. 6, pp. 418-445, 1996.

[22] B. Shirinzadeh, G. Alici, C. W. Foong, and G. Cassidy, "Fabrication process of open surfaces by robotic fiber placement," J. Robot. Computer-Integrated Manufact., vol. 20, no. 1, pp. 17-28, Feb. 2004.

[23] J. P. D. Hartog, Advanced Strength of Materials. New York: McGrawHill, 1952.

[24] M. R. Cutkosky and I. Kao, "Computing and controlling the compliance of a robotic hand," IEEE Trans. Robot. Autom., vol. 5, no. 2, pp. 151-165, Apr. 1989.

[25] H. Bruyninckx, S. Demey, and V. Kumar, "Generalized stability of compliant grasps," in Proc. IEEE Int. Conf. Robot. Autom., Leuven, Belgium, 1998, pp. 2396-2402.

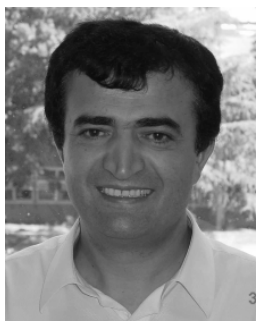

Gürsel Alici received the B.Sc. degree (with high honors) from Middle East Technical University, Gaziantep, Turkey, in 1988, the M.Sc. degree from Gaziantep University, Gaziantep, Turkey, in 1990, both in mechanical engineering, and the Ph.D degree in robotics from Oxford University, Oxford, U.K., in 1993.

He is currently a Senior Lecturer in the School of Mechanical, Materials and Mechatronics Engineering, University of Wollongong, Wollongong, NSW, Australia, where he leads Mechatronics Engineering. His research interests are in the areas of mechanics, design, control and calibration of mechanisms/robot manipulators/parallel manipulators, motion design, robotic tooling, robotics surgery and organ modeling, micro/nano robotic systems, and modeling, analysis and characterization of conducting polymer actuators.

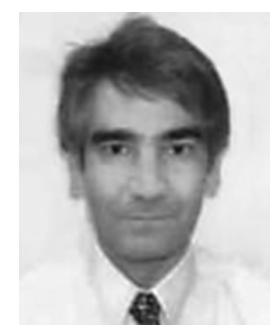

Bijan Shirinzadeh received the B.E. and M.S.E. degrees in mechanical and aerospace engineering from the University of Michigan, Ann Arbor, and the Ph.D. degree in mechanical engineering from University of Western Australia, Perth, Western Australia, Australia.

From 1990 through 1994, he was a Senior Research Scientist in Commonwealth Scientific Industrial Research Organization (CSIRO), Sydney, NSW, Australia. He is currently an Associate Professor, and the Director of Robotics \& Mechatronics Research Laboratory (RMRL) which he established in 1994 in the Department of Mechanical Engineering, Monash University, Melbourne, Victoria, Australia. His current research interests include autonomous systems, sensory-based control, laser-interferometry-based tracking and guidance, micro-nano manipulation systems, virtual reality and haptics, systems kinematics and dynamics, and automated fabrication and manufacturing. 